\title{
Dimensión económica del turismo accesible: Lecturas para Ecuador
}

\section{Economic dimension of accessible tourism: Lectures for Ecuador}

Natacha Pahola De las Mercedes Díaz Rodríguez. ${ }^{1}$, Dajhanna Pahola Carranza Díaz. ${ }^{2}$, Sergio Benjamín Carranza Mendoza. ${ }^{3} \&$ Lilia Lourdes Mora Pisco. ${ }^{4}$

Recibido: 10-11-2019/ Revisado: 05-12-209 /Aceptado: 26-12-2019/ Publicado: 04-01-2020

\begin{abstract}
.
DOI: $\underline{\text { https://doi.org/10.33262/cienciadigital.v4i1.1108 }}$

The economic dimension of accessible tourism constitutes a factor of particular importance for the development of social inclusion as a basis for sustainable human development. However, a phenomenon has been insufficiently studied in the literature and appears more as a statement than as an aspect of social relevance. The purpose of this article is to offer an analysis of the economic dimension of accessible tourism, deriving lessons for a country like Ecuador. For the analysis, the tools of qualitative research are used, based on observation and documentary analysis, which allows us to reveal, as a central idea, the importance of rethinking the practice of accessible tourism in the various regions of Ecuador as a factor of economic growth and social development, linked to the sectors of the national economy.
\end{abstract}

Keywords: Social inclusion, accessible tourism, economic conception, people with disabilities, human development.

\section{Resumen.}

La dimensión económica del turismo accesible, constituye un factor de particular importancia para el desarrollo de la inclusión social como fundamento para el desarrollo humano

\footnotetext{
${ }^{1}$ Universidad Laica Eloy Alfaro de Manabí, Ecuador, natacha.diaz@uleam.edu.ec

2 Universidad Laica Eloy Alfaro de Manabí, Ecuador, dajhapcd@ hotmail.com

${ }^{3}$ Universidad Laica Eloy Alfaro de Manabí, Ecuador, sebecame@ hotmail.com

${ }^{4}$ Universidad Laica Eloy Alfaro de Manabí, Ecuador, lilia.mora@uleam.edu.ec
} 
sostenible, sin embargo, es un fenómeno insuficientemente abordado en la literatura, el mismo aparece más como un enunciado que como un aspecto de relevancia social. A partir de esta consideración este artículo tiene como propósito, ofrecer un análisis sobre la dimensión económica del turismo accesible, derivando lecciones para un país como Ecuador. Para el análisis se emplean las herramientas de la investigación cualitativa, a partir de la observación y el análisis documental, lo que permite revelar como idea central, la importancia que tiene repensar la práctica del turismo accesible en las diversas regiones del Ecuador como un factor de crecimiento económico y desarrollo social, encadenado con los sectores de la economía nacional.

Palabras claves: Inclusión social, turismo accesible, concepción económica, personas con discapacidad, desarrollo humano.

\section{Introducción.}

El tema de la inclusión social constituye un indicador del desarrollo humano a nivel mundial, el cual es analizado en la literatura desde la visión política, económica, cultural y en su más amplio sentido de la palabra, social. En este ámbito, se promueven los estudios sobre el turismo inclusivo, término asociado a la atención diferenciada que se le otorga a las personas con discapacidad en el sector turístico, el cual ha recibido diversas denominaciones: turismo accesible, turismo inclusivo, turismo adaptado, turismo para todos, turismo sin barreras, turismo universal. Adrián, S. (2018).

El peso central de los análisis acerca del turismo accesible está enfocado desde una visión sociológica, siendo insuficientemente examinada la dimensión económica del mismo, lo cual, limita la consideración de este segmento de personas como un nicho de mercado a potenciar como factor de crecimiento económico y social. A partir de esta problemática, este artículo tiene como propósito, ofrecer un análisis sobre la dimensión económica del turismo accesible, de manera que ello contribuya a repensar su práctica en las diversas regiones y países, en lo particular para el Ecuador. Por consiguiente, situar la mirada en la creación de ciudades accesibles, a partir del impulso del turismo accesible en todos los campos de la vida cotidiana podría convertirse en un factor estratégico generador de desarrollo económico y social.

La idea anterior se fundamenta en que este segmento de discapacitados, se está convirtiendo en un demandante turístico, el cual, está creciendo en las sociedades desarrolladas, en diversas formas y tamaños según edades y dificultades de discapacidad, mercado estimado en 235 millones de dólares provenientes de la Unión Europea, Estados Unidos, Japón y Canadá; sin embargo, este potencial no se está aprovechando por la insuficiente accesibilidad, y la casi nula concepción del mismo como un nicho de mercado. Antunes Moura, A. Et, al, (2018) Tendencia a la que un país como Ecuador no escapa.

Desde una visión socio-económica es importante considerar que, no sólo hay que contar con este segmento demográfico en sí mismo, sino también con las personas sin discapacidad que los puedan acompañar. Stynes, Daniel J. (2019). La potencial incidencia del turismo accesible en la economía, 
se multiplica si se tienen en cuenta estas circunstancias. Por consiguiente, conocer las demandas de las personas discapacitadas en su conducta como turistas, permite alinear una oferta cuyos intereses estén adaptados a las mismas. Esto contribuiría a brindar mayor satisfacción a este segmento de personas y a los que los acompañan, la consecuencia sería, la generación de mayores ingresos para el país, que se podrían reinvertir en una mayor competitividad empresarial pública y privada, y de toda la cadena de valor del sector turístico. Atár, András Et, al, (2019). Ello de hecho requiere de mayor integración entre los diferentes sectores de la economía nacional.

La importancia de concebir el turismo accesible desde una concepción económica, en las diversas regiones y países está determinada por razones sociales en general, y en particular, por el hecho de que su demanda es amplia, compuesta por personas con discapacidad, que se han convertido en un segmento emergente de la demanda turística. La fundamentación de esta problemática está sustentada en que el turismo accesible contribuye al desarrollo socio-económico, cultural y ambiental, por la magnitud de este mercado debido a su potencial de crecimiento. Murillo Suárez, A. (2019) Por ello el llamado va dirigido, a la mejora de la accesibilidad de las ciudades para eliminar las barreras físicas que encuentran las personas con movilidad reducida, ello indudablemente se podría potenciar como un factor para generar mayores ingresos y empleos en las comunidades nacionales y locales, que mejoren el bienestar económico y social de los diversos sectores de la población. Kastenholz, E., et, al, (2015).

\section{Metodología}

La metodología de investigación que se aplica es la cualitativa, empleando las herramientas de la observación y análisis documental, las cuales permiten el examen del objeto de estudio en su dimensión económica, permitiendo llegar a la conclusión acerca de la importancia que tiene el turismo accesible como fundamento de estrategias socio-económicas de desarrollo con inclusión social. La norma para la presentación de citas y referencias bibliográficas empleadas es el sistema (APA).

\section{Resultados}

\section{Accesibilidad turística y turismo accesible como concreción conceptual}

Para concretar el examen que se realiza, metodológicamente resulta prudente distinguir entre accesibilidad turística y turismo accesible, lo cuales representan dos categorías socio-económicas dialécticamente interrelacionadas, que contribuyen a la integración social. COCEMFE (2018) Son condiciones de la accesibilidad en el turismo: 1) Practicar las normas instauradas, aportar y generalizar la accesibilidad entre los prestadores de servicios turísticos e organizar reglas que respalden el principio de accesibilidad, poniendo en funcionamiento procedimientos para su logro; 2) Establecer medidas de apoyo para su compensación, mejorando los procedimientos de información, de manera que llegue a todas las personas; 3) Educar e instruir a los agentes sociales de las empresas que ofrecen servicios turísticos y que interactúan con personas discapacitadas. Brielle Gillovic, et, al, (2018) 
Se infiere que la accesibilidad es transcendental para beneficiar la inclusión social considerando a todo tipo de personas, en todas las edades y de todas las condiciones. Martínez Cárdenas, R., \& Gómez Calderón, L. (2015). Por lo que el concepto de accesibilidad en el turismo económicamente hace referencia a la calidad de los servicios que se brindan en las instalaciones, posibilitando el disfrute de cualquier persona, independientemente de su capacidad física, sensorial, psíquica o cognitiva. Burgos Cortéz, E. et al, (2019).

El concepto de turismo accesible, está orientado a las actividades que se generan en establecimientos y empresas turísticas promocionan y venden la accesibilidad integral. El mismo está dirigido a una clientela de familiares y personas con discapacidad. MINCIT (2019). La consecuencia es que, económicamente el turismo accesible existe cuando la accesibilidad se ha incorporado a toda la cadena de valor del turismo, por lo que la idea es entregar accesibilidad en todos servicios, espacios y medios que forman parte del viaje y estancia del turista. Ello involucra un proceso de cambios en la infraestructura arquitectónica de una ciudad, lo cual deberá ir acompañado de cambios actitudinales de su población. Scott, M., Spyridonis, F. \& Ghinea, G. (2015).

Distinguida la accesibilidad turística y el turismo accesible, se puede plantear que este último, como concreción conceptual ha tenido diversos significados según los autores que la abordan:

- Unos autores toman como referencia a las personas con discapacidad y de la tercera edad, Scott, M., Spyridonis, F. \& Ghinea, G. (2015).

- Otros incluyen segmentos como familias con niños pequeños y personas sin discapacidad. Adrian, S. (2018)

- Unas definiciones se enfocan en como ofrecer los productos turísticos y servicios turísticos de manera universal, y otras en el disfrute de estos, y la posibilidad de participar en ellos. Fernández Alles, M. T. (2017)

La diversidad de criterios alrededor de este término permite inferir que el contenido de la categoría turismo accesible ha ido transitando, desde un contenido social a un componente que coloca la atención en el factor económico. Inicialmente el turismo accesible fue concebido para el segmento de personas con discapacidad, pero a medida que fue evolucionando en el tiempo se fue enriqueciendo situándose la mirada en su naturaleza socio-económica y cultural e introduciendo la idea de ofrecer, productos y servicios turísticos accesibles para ellos y otros segmentos de la población. Michopoulou E. et al, (2016).

La tendencia actual, es la de integrar dentro del concepto turismo accesible bajo el paraguas de un Diseño para Todos aportando factores globales como: a) La adecuación del entorno turístico para que sea accesible a todos los grupos de la población; b) Ampliación de grupos de población implicados; c) Nexo de unión entre discapacidad y personas mayores, Darcy, S. et al. (2010), d) La accesibilidad como un factor útil para el mayor número de personas posible; e) Diseño para Todos o Diseño Universal como herramienta para alcanzar la accesibilidad. Ceccarini, C. and Prandi, C. (2019) y Martínez Cárdenas, R. (2018). 
Se podría concluir parcialmente, que el concepto de turismo accesible ha evolucionado de las adaptaciones para que las personas con discapacidad puedan hacer turismo de una manera autónoma hacia un turismo de calidad para todos, entendiendo que la accesibilidad es parte importante de esta calidad humana. COCEMFE (2018)

A los efectos de la presente investigación, se asume el turismo accesible como un turismo universal orientado a personas discapacitadas, tomando como eje el disfrute del tiempo libre y bajo una concepción antropocéntrica donde el hombre forma parte primordial, siendo un factor generador de valor agregado en el sector económico. Se observa del criterio planteado, en primer lugar, el hecho de que la atención al hombre en el sentido más amplio de la palabra es esencial, y, en segundo lugar, el concepto está dirigido a una atención diferenciada de las personas con discapacidad, lo que requiere atender esta definición para una mejor comprensión del turismo accesible.

\section{Diversidad del mercado de personas con necesidades especiales es diverso}

La idea es que el tamaño del mercado de personas con discapacidad o necesidades especiales es diverso. MINCIT (2019:77). Según la Organización Mundial de la Salud, ${ }^{5}$ las personas con discapacidad, son aquellas con deficiencias físicas, mentales, intelectuales y sensoriales a largo plazo que, al interactuar con diversas barreras, puedan impedir su participación plena y efectiva en la sociedad en igualdad de condiciones con las demás. La idea que se trasmite con esta concepción, es que la discapacidad es un fenómeno que refleja una interacción entre las características del organismo humano y las características de la sociedad en la que vive. Es decir, es un fenómeno consustancial a las características del ambiente en que se desenvuelve el individuo, por lo cual requiere de tratamiento personalizado en su versión socio-económica. Según esto existen diversas manifestaciones de discapacidad según las características de las personas. (Ver Tabla No. 1)

Tabla No. 1: Tipos de discapacidad y características: Reflejo de la diversidad del mercado

\begin{tabular}{|c|c|}
\hline Discapacidad & Características \\
\hline \multirow{3}{*}{ Física } & $\begin{array}{l}\text { - Personas con movilidad reducida, dificultades en su desplazamiento o menor ca- } \\
\text { pacidad de utilizar los entornos y su relación con ellos. }\end{array}$ \\
\hline & $\begin{array}{l}\text { - Usuarios de productos de apoyo tales como: silla de ruedas, scooter, patineta eléc- } \\
\text { trica, caminadores rodantes o manuales, muletas ortopédicas, bastones y otros tipos } \\
\text { de ayuda para el desplazamiento. }\end{array}$ \\
\hline & - Personas en situación de enfermedad parcial, temporal o permanente. \\
\hline \multirow[b]{2}{*}{ Visual } & (Ceguera, baja visión, daltonismo) \\
\hline & $\begin{array}{l}\text { - Personas que poseen dificultades de visión y percepción de los espacios y entornos, } \\
\text { debido a deficiencias en las capacidades sensitivas visuales. }\end{array}$ \\
\hline
\end{tabular}

${ }^{5}$ Tomado de MINCIT (2019:77) 
- Personas con comunicación oral y auditiva nula o reducida. Su principal medio de

Auditiva comunicación es la lengua de señas / signos y lectura de labios.

- Las personas sordas, pueden tener esta condición desde o antes del nacimiento, o con posterioridad al haber aprendido alguna lengua oral.

- Personas con pérdida severa o grave de la audición y de la visión, dificultando su movilización, comunicación y participación social.

Sordo ceguera

- Según la OMS corresponde a una discapacidad múltiple y puede ser de origen congénito, adquirida con anterioridad o momento del nacimiento o en el transcurso de su vida. Esta condición podría ser acompaña de otro tipo de discapacidad.

- Personas con posible disminución y/o deficiencias por debajo del promedio, en su

Cognitiva o intelectual inteligencia y/o nivel de comunicación o capacidad de movilización.

- Persona con posibles dificultades para comprender, analizar y desarrollar destrezas para desarrollarse y movilizarse en su día a día.

- Personas con enfermedades mentales no tratadas o correctamente abordados que probablemente conducen a una discapacidad psicosocial.

Mental o Psico-social

- Personas cuyas condiciones particulares y actitudinales pueden generar barreras que impiden un adecuado desarrollo social.

- Personas con uno o más tipos de discapacidad.

Personas con discapa- - Personas que generalmente requieren acompañantes y/o personas cuidadores para cidad múltiple su acorde movilización, desarrollo personal y ejecución de actividades.

- Personas que pueden ser usuarias de perros guías y/o asistencia.

- Personas con una estatura inferior al promedio, antropométrico, de acuerdo a la edad.

Talla baja

- Personas con disminución de capacidad de movilización y desarrollo de actividades.

- Personas con disminución de capacidad de alcance y movilidad de objetos.

- Personas con una estatura superior al promedio, antropométrico, de acuerdo a la edad.

Talla alta

- Personas con una velocidad de crecimiento mayor a lo normal, desde el nacimiento hasta la primera infancia.

- Personas con posible disminución de capacidad de movilización y desarrollo en sus actividades.

- Personas con una talla, estructura y dimensión corporal más grande que el promedio de personas.

Talla gruesa

- Personas con disminución en la capacidad física o motriz.

- Personas que generalmente tienen un peso corporal superior al tamaño de sus huesos.

- Personas en situación de enfermedad, parcial o permanente, independientemente

Con situación de enferde edad, condición física, sensorial, cognitiva o mental.

medad

- Personas con requerimientos periódicos de apoyo médico y rehabilitación.

- Personas con crecimiento paulatino en la reducción de su movilidad y comunica-

Adulto mayor ción de acuerdo con las características particulares de cada persona.

- Personas con tendencia a tener una situación de enfermedad parcial o permanente.

- Personas que, por su corta edad, pueden variar su condición normal de desplaza-

Niños, niñas y jóvenes miento; saltar, correr, brincar, rodar y trepar.

- Personas que requieran de un acompañamiento, cuidador y/o perro de asistencia/guía. 
- Personas protectoras de una vida adicional dentro de su cuerpo. Estas personas en crecimiento dentro del vientre de la madre tienen sus propias necesidades y proceso Mujeres en embarazo de desarrollo independiente al de su portador.

- Las mujeres en estado de embarazo pueden tener a su cuidado uno o más niños, carros, cunas, maletines y pañaleras.

- Personas que transportan cargas adicionales y predominantes que afectan y miniDeambulantes con mizan su adecuada circulación, movilización y desplazamiento.

carga Personas que por sus cargas y elementos que movilizan, pueden afectar la estabilidad y locomoción de otras personas.

Diversidad lingüística y cultural

- Personas que viajan a un lugar donde quieran vivir experiencias diferentes.

- Personas que no tienen el adecuado conocimiento de la lengua o lenguas de determinado territorio y/o país.

Fuente: Elaboración propia sobre la base de MINCIT (2019)

De la Tabla No. 1 se puede extraer como conclusión parcial que el tamaño del mercado de personas con necesidades especiales es diverso por la variedad de segmentos existentes, lo cual requiere de atenciones especiales. Los datos siguientes son notables: (Ver gráfico No. 1)

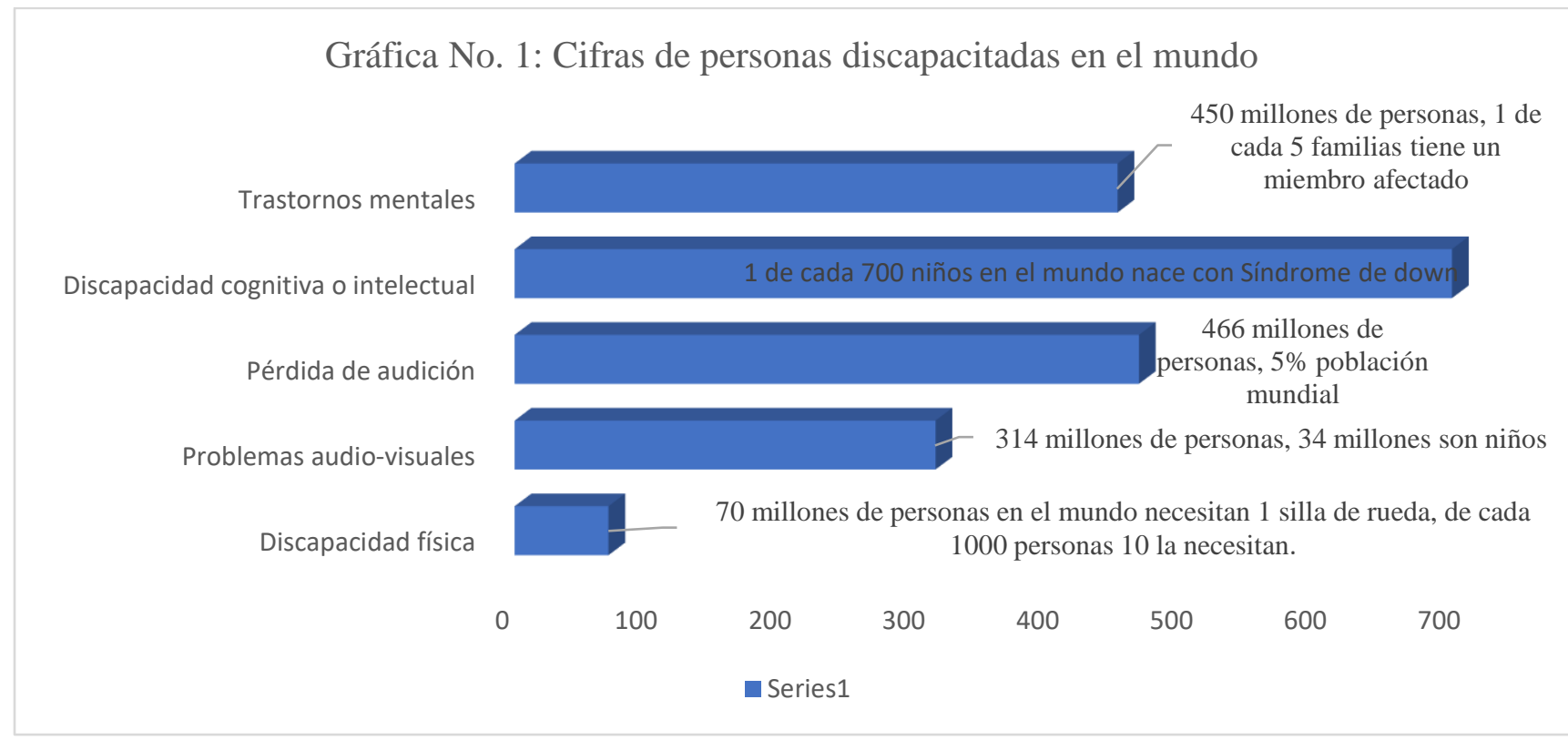

Fuente: Elaboración propia sobre la base de MINCIT (2019:21-25)

Los datos del gráfico No. 1 son expresión de la gravedad del fenómeno creciente de personas discapacitadas, lo cual requiere de tratamientos individualizados en relación a su acceso a servicios 
sociales, en lo que el desarrollo del turismo accesible pudiera contribuir de manera importante. A dichas cifras se pueden añadir otras, que también son preocupantes:

"Según la Organización mundial de la Salud (OMS), en el informe 2015 sobre el envejecimiento y salud: el número de personas de 60 años se duplicaría entre el 2000 y 2050, pasando del $11 \%$ al 22\%, este grupo de edad pasará de 605 millones a 2000 millones en el transcurso de medio siglo. Por otro lado, los estudios estiman que en el mundo por cada 25000 personas nace 1 con alteraciones genéticas que producen la acondroplasia (personas de baja estatura); UNICEF indica que en el mundo hay 2100 millones de niños y niñas, que representan un $36 \%$ de la población. Todos los años nacen alrededor de 132 millones de niños y niñas"6

Claro que los datos antes citados, por sí solos no indican las posibilidades de este segmento de personas para beneficiarse del turismo accesible, debido a las profundas desigualdades sociales existentes en el mundo actual como consecuencia de las limitaciones socio-económicas de los sistemas sociales vigentes Sin embargo, son una evidencia de la variedad de personas que se podrían incorporar a partir de sus potencialidades como un segmento de mercado para su inclusión social en las perspectivas del turismo accesible.

\section{Negocio del siglo XXI}

La magnitud y variedad del segmento poblacional con discapacidad ha hecho que se etiquete la frase de que el desarrollo del turismo accesible es el "NEGOCIO DEL SIGLO XXI". Stergiou, D. and Airey, D. (2018). Esto está basado en el hecho de que un sistema hotelero independientemente de su tamaño, localización, y características del servicio, marca la diferencia, y da un valor agregado, solo por contar con servicio de transporte, playa accesible, menú del restaurante, en sistema braille y atención en lengua de señas / signos. Ello marca la diferencia con relación al turismo convencional, al ampliarse las posibilidades de generar mayores ingresos por la diversidad de servicios prestados. El hecho es que las ventajas competitivas del desarrollo del turismo accesible van a ser variadas: MINCIT (2019:27-28) y Kołodziejczak, A. (2019).

a. Aumento de mercado: El mercado potencial de personas viajeras se aumenta entre el 15 y $20 \%$, normalmente viajan acompañados, por lo que son multicliente;

b. Incrementa la rentabilidad: Requieren de diversos servicios y atenciones por lo que, a mayor cantidad de viajeros, mayor generación de ingresos en el turismo;

c. Reducir y destacionalizar: Viajan en cualquier época del año, favoreciendo la desestacionalidad;

d. Aumento de usuarios: Se estima que una persona con discapacidad o en situación de enfermedad incrementa el número de acompañantes de viaje;

e. Reconocimiento e imagen: Un espacio accesible aumenta la favorabilidad en la imagen positiva e incluyente de un país, región o ciudad en cualquier parte del mundo;

\footnotetext{
${ }^{6}$ MINCIT (2019:21-25)
} 
f. Disminuye la competencia en los diferentes mercados;

g. Aumenta la innovación y tecnología de punta, en los diferentes entornos, servicios, procesos, espacios y productos.

\section{Lecturas para Ecuador: Enganchar el desarrollo accesible al cambio de la matriz productiva}

Para el caso de Ecuador como lección se recomienda orientar las estrategias de desarrollo de turismo accesible enganchadas del cambio de la matriz productiva, lo cual constituye una prioridad de la política económica del país, para enfrentar la estructura tradicional basada en la generación de ingresos a partir de productos de la pesca y el petróleo. Díaz Rodríguez, N. y Mora Pisco, L. Durán, M. (2019).

La idea es potenciar el turismo accesible, en un espacio donde el turismo actualmente ocupa la tercera fuente de ingresos de la nación. ${ }^{7}$ En este escenario se podría insertar la mirada de la creación de ciudades accesibles en las diversas regiones de Ecuador. Ello beneficiaría socialmente a la población local, en un país donde el 3,5 \% de la población (15.952.442) - según el Instituto Nacional de Estadística y Censos (INEC)- presenta algún tipo de discapacidad, distribuidos de la manera siguiente: Ver gráfico No. 2.

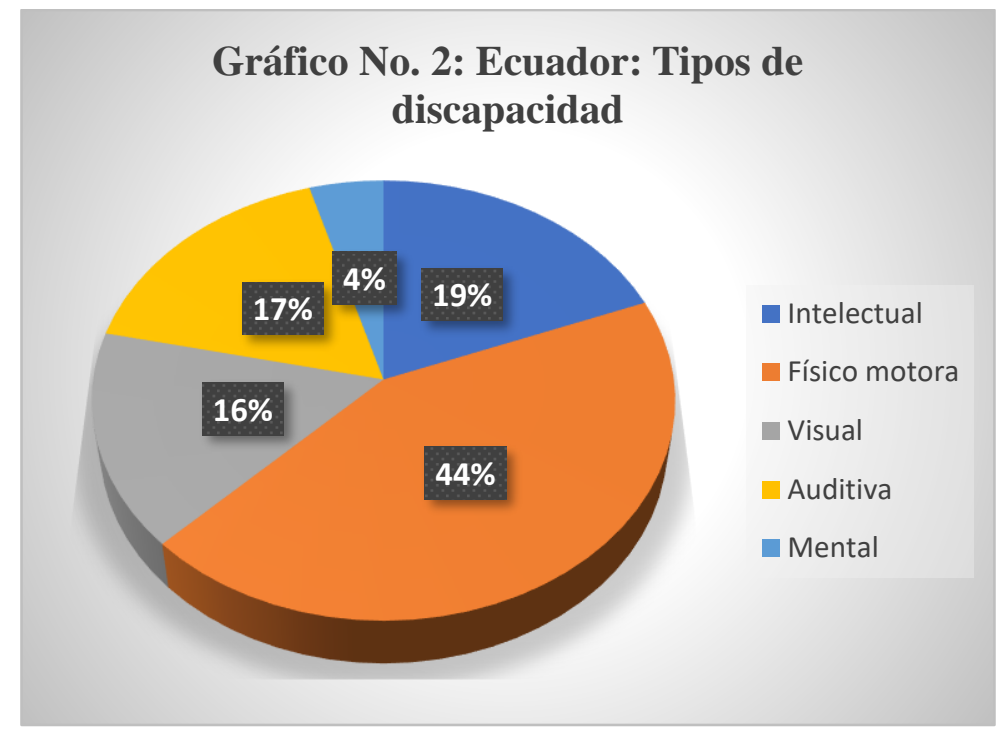

Fuente: Elaboración propia, sobre la base de datos del INEC. Periodo 2013-2014

Para Ecuador las lecturas de desarrollar el turismo accesible serían múltiples, sobre todo por los beneficios que se pudieran esperar de ello: Mejora de la accesibilidad turística desde los servicios públicos y privados supone inclusión social; podría ser un factor para la consolidación del marco legislativo y normativo como garantía de un derecho fundamental del ciudadano en las diversas

\footnotetext{
${ }^{7}$ Según la Cuenta de Viajes del Banco Central del Ecuador, (2018) los ingresos de divisas por turismo, entre el 2013 y 2017 crecieron en un $7 \%$, ubicándose en el año 2017, en el tercer lugar de ingreso de divisas entre los bienes y servicios no petroleros ${ }^{7}$ en el contexto de la economía nacional, con 1.633 millones de USD, después del banano y el camarón.
} 
regiones. De ello se deriva, la plena integración de un emergente segmento de mercado turístico: el de las personas con discapacidad, que crece a pasos galopantes a nivel mundial.

Por ejemplo, en este entorno social, la población de la tercera edad va en aumento exigiendo servicios turísticos especializados con fines de disfrute. Esta tendencia ya está exigiendo un mayor reordenamiento del componente económico y social con integración hacia adelante y hacia atrás en los diversos productos y servicios que se ofertan, lo que implica para los diversos sectores económicos asociados al turismo, potenciar una cultura económica articulada desde lo social, basada en la personalización del cliente y en el desarrollo de un inminente proceso de encadenamientos productivos, donde la industria nacional se nutra de los recursos internos del país, contribuyendo a la sustitución de importaciones.

Según lo anterior, se espera que el impacto del crecimiento de las demandas del turismo accesible en la conduzca a un perfeccionamiento de los vínculos de los diversos actores que componen la cadena de valor del turismo, dígase las relaciones entre los diversos entes públicos, entre los privados y las relaciones de las entidades turísticas públicas y privadas. Es decir, que, en materia de gestión empresarial, deberá producirse un cambio en la manera de conducir la actividad turística de la región, teniendo en cuenta las exigencias del turismo accesible.

La idea es que la accesibilidad en su dimensión económica, no se resuelve sólo con incorporar herramientas tecnológicas para el acceso al comercio, restaurante u hotel, es un concepto que involucra modos de actuación diferentes, sensibilización, concienciación e involucramiento personal, laboral y empresarial más amplia en el sentido social y económico, es un beneficio para todos que tiene su sustento en el bien común.

Por consiguiente, la accesibilidad, la integración social y el turismo accesible deberá, además, ser fruto de una formación profesional que es necesario ampliar y mejorar. En este sentido, para el sector turístico es un desafío, que supone desde lo socio-económico, orientar el personal, a la prestación de servicios especializados, lo cual involucra la atención al público con todas sus manifestaciones particulares de diversidad.

Considerando el lado de las utilidades que podría producir el turismo accesible como oportunidad de negocios las cifras -antes citadas- indican que las personas discapacitadas o de movilidad reducida, generalmente viajan con uno o dos acompañantes, lo cual tiene un efecto multiplicador desde este segmento de demanda, para las empresas públicas, privadas y actores locales. El efecto es que el impacto socio-económico de la promoción de este segmento de mercado, podría ser exitoso, por lo que significa para la generación de ingresos, empleos, nuevas inversiones e infraestructuras. Se espera que, de seguir las tendencias mundiales de este nicho de negocio, y su aplicación práctica para atraer turistas de este segmento de mercado, se concreten muchos sueños de ciudades accesibles.

La realización del ejercicio de reflexión sobre el impacto esperado de un programa para el desarrollo de turismo accesible sugiere, además, considerar los efectos inducidos de una mayor población de turistas que requieren servicios especiales. Ello induce una mayor cantidad de dinero 
en la circulación al producir una mayor cantidad de empleos, lo que mejoraría los ingresos de los hogares en los ingresos obtenidos directa o indirectamente como resultado del gasto turístico. Una muestra de ello, son los empleados de los diversos sectores de apoyo al turismo, quienes realizan sus operaciones de gastos de sus ingresos en la región en actividades de mejora de sus salud, transporte, educación, alimentación y vivienda, así los efectos directos, indirectos e inducidos de estos, tendrán un efecto multiplicador para todos los sectores de la economía local.

Esto supone el desarrollo de ofertas de productos y servicios para la población y empresas locales, que estimulen la propensión a invertir sus recursos a proveedores del territorio, sugiere, además, estimular una mayor estadía del turista en la ciudad, lo cual induce mayores gastos que beneficiarían a las empresas y hogares de la localidad.

Dichos ingresos resultantes del desarrollo del turismo accesible, invertidos de manera eficiente y eficaz podrían orientarse a la capacitación de profesionales; a la creación de nuevas infraestructuras turísticas; al mejoramiento de los servicios hospitalarios y de educación del país; al desarrollo de proyectos que garanticen la soberanía tecnológica y de la producción de alimentos; y a la puesta en práctica de vínculos con capital extranjero, que aporte capital fresco para el desarrollo endógeno, promoviendo mayor valor agregado a las ofertas de productos para el consumo nacional y para la exportación, a partir de la idea de que dicho vínculo, se sustente con insumos nacionales y no con consumo de productos importados, teniendo como base que la inversión extranjera esté encadenada a los sectores de la economía nacional.

La resultante de lo anterior sería un tipo de turismo accesible que tiene la capacidad de promover el crecimiento económico inclusivo contribuyendo a un desarrollo más equitativo, a partir de la redistribución de los dividendos del crecimiento entre los individuos y los grupos sociales, incluido el aumento del bienestar, que está moldeado por dimensiones no materiales, como el estado de la salud y la educación, y el medio ambiente.

Para el caso, de las zonas urbanas, con el desarrollo del turismo accesible se espera que promueva una cultura de servicios que involucre a todos los pobladores locales, bajo la guía del sector público y privado. La expectativa es, además, que la promoción y concreción de este segmento de mercado, contribuya a generar financiamiento proveniente de empresas nacionales y extranjeras.

Con el impacto socio-económico de promover turismo accesible se podrían ampliar las zonas rurales a través de cadenas de suministro para satisfacer la creciente demanda de productos de producción local como gastronomía, vino, arte y artesanía. En las regiones rurales, el turismo accesible podría convertirse en una palanca para el desarrollo y el crecimiento económico, articulada en torno a la agricultura, la cultura y las actividades al aire libre. No se trata de crear una oferta exclusiva para personas con discapacidad, ya que este no es un nicho de mercado que requiera un producto específico. La realidad pasa por ampliar la misma oferta existente, con el objetivo de llegar a todas les personas, independientemente de sus limitaciones personales.

La mejora de la accesibilidad a los equipamientos, recursos y servicios contribuiría a eliminar las desigualdades/igualdad de oportunidades en el acceso al turismo, permitiendo el uso y disfrute de 
la oferta y servicios turísticos a personas con discapacidad y/o con movilidad reducida. Esto incrementa la calidad de vida de este segmento de personas, teniendo que hacer un menor esfuerzo para realizar sus actividades ocio, traslado, hospedaje y restauración en el destino. La no accesibilidad turística es una forma de discriminación indirecta, ya que genera una desventaja real entre personas con alguna discapacidad y personas no tienen.

\section{Discusión}

La clave del desarrollo de turismo accesible actualmente, es desarrollarlo bajo una concepción económica que involucre todos los entes sociales que participan como agentes en esta dimensión social, dígase el Estado, las empresas públicas y privadas y los actores de la comunidades, es esta la idea central en que avanza la presente investigación en relación a otros autores. ${ }^{8}$ El pensamiento central del presente artículo, va dirigido a favorecer las fortalezas de las localidades, regiones y países desde la cultura del turismo, aprovechando las oportunidades de un mercado creciente como el de las personas discapacitadas como factor de desarrollo económico y social.

\section{Conclusiones.}

- La importancia del estudio del turismo accesible en su dimensión económica, no sólo radica por el elevado número de personas que lo integran y por su creciente participación en el mercado turístico, sino también por su futuro potencial de crecimiento, todo lo cual ha llevado a su consideración como oportunidad de negocio para el sector turístico.

- Uno de los segmentos de mercado que mayor protagonismo que ha cobrado en los últimos años es el de las personas discapacitadas. Su repercusión en múltiples ámbitos de la economía, la política, la cultura y la sociedad en su conjunto, se ha hecho especialmente patente en el sector turismo, por lo que la relevancia de este segmento de mercado para el mismo, viene justificada por dos razones fundamentales: el número de efectivos que lo integran, así como su participación cada vez mayor en el mercado turístico.

- Las experiencias internacionales sobre el tema orientan la atención sobre las líneas a definir, y trabajar, para incorporar el pensamiento sobre turismo accesible, al sistema de políticas nacionales y locales como factor de crecimiento económico y social, que tiene como principio básico la inclusión social.

- Los resultados esperados de una propuesta de desarrollo de la dimensión económica del turismo accesible prevé siempre cambios en los diversos ámbitos del desarrollo social, en la política turística, favoreciendo encadenamientos empresariales, modificaciones en el turismo urbano y rural, con impactos en una mayor cantidad de empleos mejor retribuidos, el crecimiento de la rentabilidad del sector público y privados, que deberá favorecer a las comunidades locales y a los diversos sectores de la cadena del sector turístico.

- Un país como el Ecuador, con el potencial de sus recursos naturales y humanos tiene el reto de repensar la configuración del desarrollo del turismo accesible encadenado al cambio de la matriz productiva, aprovechando la demanda creciente del sector de discapacitados a

${ }^{8}$ Burgos Cortéz, E. et al, (2019), Fernández Alles, M. T. (2017, Michopoulou E. Darcy S. Ambrose I. Buhalis D. 2016, Kastenholz, E., et, al, (2015). 
nivel mundial, lo cual favorecería a los pobladores locales del país que padecen de discapacidades y a la población ecuatoriana en general. La idea es promover la integración de la economía productiva a nivel de país desde la mirada del turismo accesible.

\section{Referencias Bibliográficas.}

Adrian, S. (2018). ¿Qué es un espacio inclusivo? Disponible en: http://parquesalegres.org/biblioteca/blog/que-es-un-espacio-inclusivo/.

Antunes Moura, A. Et, al, (2018) Accessible tourism and its benefits for coping with stress, Journal of Policy Research in Tourism, Leisure and Events, 10:3, 241-264, DOI: 10.1080/19407963.2017.1409750.

Atár, András Et, al, (2019) Accessible tourism services in the North Great Plain region = Hozzáférhetö turisztikai lehetöségek az észak-alföldi régióban. Különleges Bánásmód, 5 (1). pp. 77-81. ISSN 2498-5368.

Brielle Gillovic, et, al, (2018) Enabling the language of accessible tourism, Journal of Sustainable Tourism, 26:4, 615-630, DOI: 10.1080/09669582.2017.1377209.

Burgos Cortéz, E. et al, (2019). Influencia de la gestión de la Red de Turismo Accesible del Ecuador (REDTAEC) en Baños de Agua Santa. Carrera de Ingeniería en Administración Turística y Hotelera. Universidad de las Fuerzas Armadas ESPE. Matriz Sangolquí.

Ceccarini, C. and Prandi, C. (2019). "Tourism for all: a mobile application to assist visually impaired users in enjoying tourist services," 2019 16th IEEE Annual Consumer Communications \& Networking Conference (CCNC), Las Vegas, NV, USA, 2019, pp. 16. doi: 10.1109/CCNC.2019.8651848.

COCEMFE (2018). Breve Historia de la Accesibilidad. Disponible en: https://www.observatoriodelaaccesibilidad.es/accesibilidad/breve-historia/.

Darcy, S. et al. (2010), 'Accessible tourism and sustainability: A discussion and case study', Journal of Sustainable Tourism.

Díaz Rodríguez, N. y Mora Pisco, L. Durán, M. (2019). Las bases del cambio de la matriz productiva en Ecuador (2006-2016). Revista Científica de la Universidad de Cienfuegos. Volumen 11, Número 4, Julio-Septiembre 2019. Universidad y Sociedad, Cuba.

Fernández Alles, M. T. (2017). Turismo Accesible: Importancia de la Accesibilidad para el Sector Turístico, (May).

Kastenholz, E., et, al, (2015). Contributions of tourism to social inclusion of persons with disability. Disability and Society, 30(8), 1259-1281. Disponible en: https://doi.org/10.1080/09687599.2015.1075868.

Kołodziejczak, A. (2019). Information as a Factor of the Development of Accessible Tourism for People with Disabilities. Volume 38: Issue 2. Disponible en: https://content.sciendo.com/view/journals/quageo/38/2/articlep67.xml?tab_body=contentReferences. 
Martínez Cárdenas, R., \& Gómez Calderón, L. (2015). Turismo accesible, una alternativa de inclusión social y equidad. In Espaces et environnements littoraux et insulaires. Accessibilité Vulnérabilité-Résilence. París, Francia: EDITIONS KARTHALA.

Michopoulou E. Darcy S. Ambrose I. Buhalis D. 2016. Accessible tourism futures: The world we dream to live in and opportunities we hope to have. Journal of Tourism Futures 1(13): 179188.

MINCIT (2019). Manual de turismo accesible "Turismo para Todos". Autor: Ministerio de Comercio, Industria y Turismo (MINCIT) Fondo Nacional de Turismo (FONTUR) Consejo Iberoamericano de Diseño, Ciudad y Construcción Accesible (CIDCCA), Edición: Primera, Publicación: Bogotá, Colombia.

Murillo Suárez, A. (2019). Análisis de accesibilidad de la Parroquia Rocafuerte para el diseño de un recorrido turístico direccionado a discapacitados visuales, cantón Guayaquil. Tesis para optar por el título de Licenciado en Hotelería y Turismo. UNIVERSIDAD DE GUAYAQUIL FACULTAD DE COMUNICACIÓN SOCIAL CARRERA DE TURISMO Y HOTELERÍA.

Scott, M., Spyridonis, F. \& Ghinea, G. (2015). Designing for de-signers: Towards the development of accessible ICT products and services using the VERITAS framework. Computer Standards \& Interfaces, 42, 113-124. http://doi.org/10.1016/j.csi.2015.05.004.

Stergiou, D. and Airey, D. (2018), "Understandings of tourism theory", Tourism Review, Vol. 73 No. 2, pp. 156-168. https://doi.org/10.1108/TR-07-2017-0120.

Stynes, Daniel J. (2019). Economic Impacts of Tourism. Disponible en: https://msu.edu/course/prr/840/econimpact/pdf/ecimpvol1.pdf.

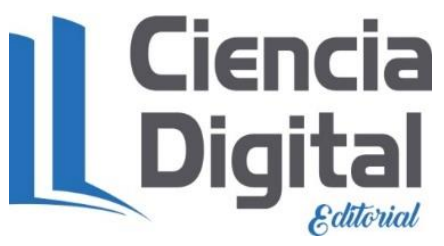


Vol. 4, $\mathrm{N}^{\circ} 1$, p. 334-348, enero - marzo, 2020

\section{PARA CITAR EL ARTÍCULO INDEXADO.}

Díaz Rodríguez, N. P. D. las M., Carranza Díaz, D. P., Carranza Mendoza, S. B., \& Mora Pisco, L. L. (2020). Dimensión económica del turismo accesible: Lecturas para Ecuador. Ciencia Digital, 4(1), 334348. https://doi.org/10.33262/cienciadigital.v4i1.1108

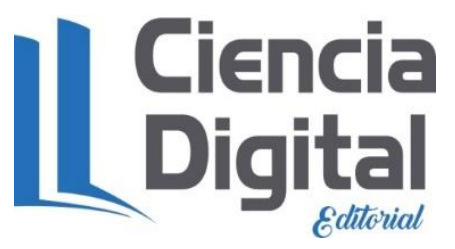

El artículo que se publica es de exclusiva responsabilidad de los autores y no necesariamente reflejan el pensamiento de la Revista Ciencia Digital.

El artículo queda en propiedad de la revista y, por tanto, su publicación parcial y/o total en otro medio tiene que ser autorizado por el director de la Revista Ciencia Digital.
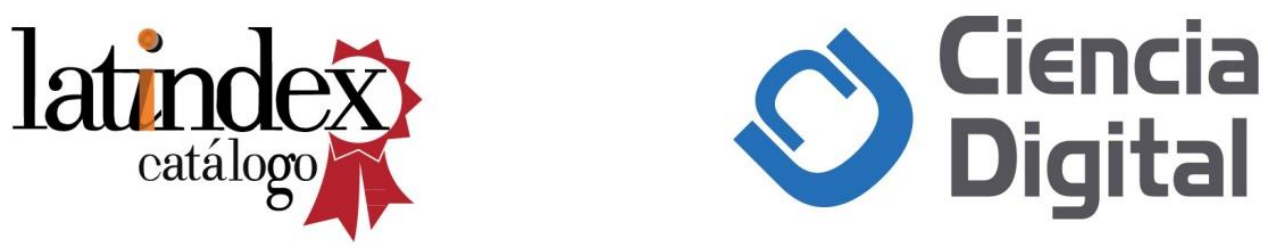